
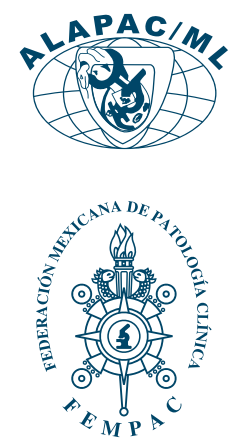

Palabras clave: Seguridad del paciente, laboratorio clínico, valores críticos

Keywords:

Patient safety, clinical laboratory services, critical values.

\section{* Servicio de Análisis Clínicos. Hospital Universitario de Móstoles. Madrid, España. \\ ¥ Servicio de Análisis Clínicos. Hospital Universitario Rio Hortega. Valladolid. Castilla y León, España, Profesor del Master de Seguridad Clínica del Paciente y de la Calidad Asistencial. UNIR.}

Correspondencia: Laura Criado Gómez Farmacéutica Especialista en Análisis Clínicos.

Calle Bustamante Núm. 3, 4º B, 28045, Madrid. Tel: 626813975 E-mail: Icriadog@ salud.madrid.org

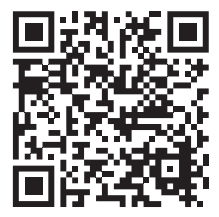

\title{
Uso de valores críticos como herramienta fundamental en la seguridad del paciente por el laboratorio
}

\author{
Use of critical values as a fundamental tool in the \\ safety of the patient by the laboratory
}

Criado Gómez Laura,* Villanueva Curto Santiago,*

Olmos Sánchez Isabel Clara,* Paniagua Arribas Esther,* García García Carmen,*

Reig Del Moral Jorge,* San Miguel Hernández Ángel ${ }^{\ddagger}$

\section{RESUMEN}

Introducción: La seguridad del paciente es de vital importancia en el ámbito sanitario, en el que se incluye el laboratorio clínico. El aviso de valores críticos forma parte de los procesos postanalíticos, y aparece en los objetivos estratégicos de los Planes de Seguridad del Paciente en todas las comunidades autónomas. El objetivo de nuestro estudio fue mejorar la estrategia de aviso de valores críticos mediante la actualización del protocolo existente a través del consenso con los clínicos, a la vez que fomentar la cultura de seguridad del paciente en la organización. Material y métodos: Se difundieron encuestas vía email a los clínicos de atención especializada y atención primaria para realizar el protocolo consensuado con ellos. Resultados: Se obtuvieron 57 respuestas en general de todas las especialidades, se desconoce a cuántos clínicos llegó, pero consideramos que fue un bajo número de respuestas, lo que denota falta de implicación en la seguridad del paciente. Conclusiones: Se actualizó el protocolo con los nuevos valores, entre los que destacan cambios en los niveles de hiponatremia, hipocalcemia, creatinina, y creatininquinasa (CK). La implementación del nuevo protocolo supondrá mejoras en la seguridad del paciente en el laboratorio clínico.

\section{ABSTRACT}

Introduction: Patient safety is of vital importance in the health sector, which includes the clinical laboratory. Notice of critical values is part of the post analytical process and it appears within the strategic objectives of the Patient Safety Plans in all the Autonomous Communities. The aim of our study was to perform an update to our existing protocol by consensus with the clinicians. In addition promoting a culture of patient safety in the organization. Material and methods: For this, we carry out surveys for email to primary and specialized care clinicians with differents analytes and values, and how and who warn whenever there are. Results: 57 responses were obtained in surveys, in general of the all especialities. We know how many people I get, but we consider low number of answers, that translates into lack of involvement in safety culture. Conclusions: Updated the protocol with the new values, including changes in the levels of hyponatremia and hypocalcemia, creatinine, and creatininquinasa. The implementation of the new protocol will result improvements in the patient safety in the clinical laboratory.

\section{INTRODUCCIÓN}

$-1$ I estudio ENEAS (Estudio Nacional sobre los Efectos Adversos Ligados a la Hospitalización) estimó que $2.74 \%$ de los errores rela- cionados con la seguridad del paciente tenían que ver con el diagnóstico, dentro de ellos se sitúan los relacionados con el laboratorio clínico, esto es algo que ponen de manifiesto muchas organizaciones internacionales. ${ }^{1}$

Citar como: Criado GL, Villanueva CS, Olmos SIC, Paniagua AE, García GC, Reig MJ, San Miguel HA. Uso de valores críticos como herramienta fundamental en la seguridad del paciente por el laboratorio. Rev Mex Patol Clin Med Lab. 2020; 67 (2): 69-75. doi: 10.35366/95549 
Se estima que $70 \%$ de los errores del laboratorio ocurre en la fase preanalítica y $15 \%$ en la postanalíti$\mathrm{ca}^{2,3}$ dentro de la fase postanalítica, uno de los aspectos prioritarios, y muy susceptible a errores, es el aviso o la comunicación de los valores críticos. ${ }^{4,5}$

La definición de valor crítico fue acuñada por Lundberg $^{6}$ en 1972: son los resultados de pruebas diagnósticas que expresan una situación médica que puede poner en riesgo la vida del paciente si no se interviene adecuada y oportunamente, y de ahí la importancia de que sean comunicados al médico solicitante de la analítica de manera inmediata. Es muy importante no llegar a confundir valor crítico con valor de alarma. ${ }^{7}$ En el año 2015, Clinical and Laboratory Standards Institute (CLSI) publicó la guía GP47 Management of Criticaland Significant-Risk Results donde recomienda usar los conceptos «resultado con riesgo crítico» y «resultado con riesgo significativo» respectivamente para valor crítico y de alarma. ${ }^{8}$ La Organización Mundial de la Salud (OMS) y la Joint Comission on Accreditation of Healthcare Organizations (JCAHO) (en el marco de la acreditación de los laboratorios clínicos estadounidenses) también hacen referencia a la importancia de la repercusión que los valores críticos tienen en la seguridad del paciente ${ }^{9}$ y lo valioso que es para ello contar con líderes dentro de la organización que la promuevan.

El aviso de valores críticos es de obligado cumplimiento para todos aquellos laboratorios acreditados por la norma UNE EN ISO 15189, dentro del subapartado 5.8.7 se indica que «el laboratorio debe tener procedimientos para avisar inmediatamente cuando los resultados de los análisis correspondientes a propiedades críticas se encuentren dentro de los intervalos de alarma establecidos» y dentro del subapartado 5.8.8 se indica que «el laboratorio debe definir las propiedades cuyos valores pueden ser alarmantes y los intervalos correspondientes de acuerdo con los médicos clínicos» ${ }^{10}$ En la actualidad los laboratorios, aunque no estén acreditados, suelen tener un protocolo de aviso de valores críticos dada la importancia y repercusión del tema.

Aunque el término «valor crítico» es antiguo, a diferencia del término «seguridad del paciente», continúa siendo emergente hoy en día. No existen recomendaciones nacionales ni internacionales sobre el adecuado procedimiento del aviso de valores críticos. Se ha escrito mucho al respecto y finalmente las directrices van todas en la misma dirección: cada laboratorio deberá realizar su propia estrategia, consensuando los valores con los clíni$\cos ^{11}$ así como la persona a quien se avisa, el medio y el tiempo de aviso. ${ }^{4,7,12-14}$ Se han realizado muchos estudios a través de encuestas, de alguno de ellos se desprenden directrices: ${ }^{13}$ los valores deben ser verdaderamente críticos (la vida del paciente está en juego y requiere la actuación inmediata por parte del clínico), deben referirse a edad y género, y deben tener efectos sustanciales en la seguridad del paciente. Este último punto es el más controvertido, puesto que no hay suficientes estudios que demuestren el efecto positivo o negativo que el aviso de los valores críticos tiene en los pacientes; ${ }^{12,13}$ pese a la escasa evidencia científica es algo que hoy en día no se pone en tela de juicio.

Es importante, y así lo recoge la norma UNE EN ISO $15189,{ }^{10}$ fijar en el protocolo cómo y a quién se debe avisar. Hay estudios que demuestran que lo más rápido es el aviso telefónico; ${ }^{14}$ sin embargo, hay que asegurarse de que el interlocutor lo ha entendido (read-back), o en su defecto que conste también por escrito, evitando errores de información verbal, ${ }^{15-17}$ y que la persona a la que se comunique la información debe tener capacidad suficiente de discernir y tomar una decisión al respecto del paciente.

La formación y el liderazgo serán elementos imprescindibles para llevar a cabo la estrategia, ${ }^{3}$ y la cultura de seguridad del paciente mejorará dentro y extensiblemente fuera del laboratorio. Debemos resaltar que dentro de la estrategia de seguridad del paciente del Servicio Madrileño de Salud 2015-2020 aparece la comunicación de valores críticos como uno de sus objetivos.

Por todo ello establecemos los siguientes objetivos para nuestro trabajo:

\section{Objetivo principal}

Mejora de la estrategia de aviso de valores críticos mediante la actualización del protocolo del servicio de análisis clínicos del laboratorio programado a través consenso con los clínicos.

\section{Objetivos secundarios}

- Establecer indicadores para monitorizar la estrategia de aviso de valores críticos implantada.

- Fomentar la cultura de la seguridad del paciente en el propio servicio de análisis clínicos y en la organización en clínicos de atención especializada y atención primaria.

\section{MATERIAL Y MÉTODOS}

Nuestro hospital es un centro público que da cobertura a una población de 150,000 habitantes. Alberga 300 camas de hospitalización, y presenta labores asistenciales, inves- 
Tabla 1: Tabla de aviso de valores críticos previos y tras la instauración del nuevo protocolo.

\begin{tabular}{|c|c|c|}
\hline & Valor crítico previo & Nuevo valor crítico \\
\hline Ácido úrico & $>13 \mathrm{mg} / \mathrm{dL}$ & $>13 \mathrm{mg} / \mathrm{dL}$ \\
\hline $\begin{array}{l}\text { Aminotrans- } \\
\text { ferasas }\end{array}$ & $>1,000 \mathrm{U} / \mathrm{L}$ & $\begin{array}{c}>1,000 \mathrm{U} / \mathrm{L} \\
\text { (adultos) } \\
>100 \mathrm{U} / \mathrm{L} \\
\text { (menores } 14 \text { años) }\end{array}$ \\
\hline $\begin{array}{l}\text { Bilirrubina } \\
\text { total }\end{array}$ & $\begin{array}{c}>15 \mathrm{mg} / \mathrm{dL} \\
(257 \mathrm{mmol} / \mathrm{L})\end{array}$ & $>15 \mathrm{mg} / \mathrm{dL}$ \\
\hline \multirow[t]{2}{*}{ Calcio, total } & $\begin{array}{c}<6.6 \mathrm{mg} / \mathrm{dL} \\
(1.65 \mathrm{mmol} / \mathrm{L})\end{array}$ & $<6.5 \mathrm{mg} / \mathrm{dL}$ \\
\hline & $\begin{array}{l}>14 \mathrm{mg} / \mathrm{dL} \\
(3.5 \mathrm{mmol} / \mathrm{L})\end{array}$ & $>13.5 \mathrm{mg} / \mathrm{dL}$ \\
\hline Creatinina & $>7.4 \mathrm{mg} / \mathrm{dl}$ & $>5 \mathrm{mg} / \mathrm{dL}$ \\
\hline Ck & $>1,000 \mathrm{U} / \mathrm{L}$ & $>1,500 \mathrm{U} / \mathrm{L}$ \\
\hline Digoxina & $>2.0 \mathrm{ng} / \mathrm{mL}$ & $>2.0 \mathrm{ng} / \mathrm{mL}$ \\
\hline \multirow[t]{2}{*}{ Fósforo } & $\begin{array}{l}<1.0 \mathrm{mg} / \mathrm{dL} \\
(0.32 \mathrm{mmol} / \mathrm{L})\end{array}$ & $<1.0 \mathrm{mg} / \mathrm{dL}$ \\
\hline & $\begin{array}{l}>9.0 \mathrm{mg} / \mathrm{dL} \\
(2.9 \mathrm{mmol} / \mathrm{L})\end{array}$ & $>8.5 \mathrm{mg} / \mathrm{dL}$ \\
\hline \multirow[t]{2}{*}{ Glucosa } & $<45 \mathrm{mg} / \mathrm{dL}$ & $<45 \mathrm{mg} / \mathrm{dL}$ \\
\hline & $>500 \mathrm{mg} / \mathrm{dL}$ & $>400 \mathrm{mg} / \mathrm{dL}$ \\
\hline LDH & $>1,000 \mathrm{U} / \mathrm{L}$ & $>1,000 \mathrm{U} / \mathrm{L}$ \\
\hline \multirow[t]{2}{*}{ Magnesio } & $<1 \mathrm{mg} / \mathrm{dL}$ & $<1 \mathrm{mg} / \mathrm{dL}$ \\
\hline & $>4.9 \mathrm{mg} / \mathrm{dL}$ & $>4.9 \mathrm{mg} / \mathrm{dL}$ \\
\hline \multirow[t]{2}{*}{ Potasio } & $<2.8 \mathrm{mmol} / \mathrm{L}$ & $<2.8 \mathrm{mEq} / \mathrm{L}$ \\
\hline & $>6.2 \mathrm{mmol} / \mathrm{L}$ & $>6.5 \mathrm{mEq} / \mathrm{L}$ \\
\hline \multirow[t]{2}{*}{ Sodio } & $<120 \mathrm{mmol}$ & $<125 \mathrm{mEq} / \mathrm{L}$ \\
\hline & $>160 \mathrm{mmol} / \mathrm{L}$ & $>160 \mathrm{mEq} / \mathrm{L}$ \\
\hline Troponina & $>0.1 \mathrm{mg} / \mathrm{L}$ & $>50 \mathrm{pg} / \mathrm{mL}$ \\
\hline Urea & $>214 \mathrm{mg} / \mathrm{dL}$ & $>200 \mathrm{mg} / \mathrm{dl}$ \\
\hline TSH & & $\begin{aligned} &> 50 \mathrm{uUl} / \mathrm{mL} \\
& \text { (adultos) } \\
&> 10 \mathrm{uUl} / \mathrm{mL} \\
& \text { (< } 14 \text { años) }\end{aligned}$ \\
\hline
\end{tabular}

tigadores y docentes. En la actualidad es un hospital de segundo nivel con una elevada carga asistencial. La atención especializada comprende actividades asistenciales, diagnósticas, terapéuticas y de rehabilitación y cuidados así como aquéllas de promoción de la salud, educación sanitaria y prevención de la enfermedad, cuya naturaleza aconseja que se realicen en este nivel.

El laboratorio de análisis clínicos consta de un laboratorio de urgencias (que no será objeto del presente trabajo) y un laboratorio de rutina programado, donde se reciben analíticas de pacientes ingresados y pacientes ambulantes procedentes de las consultas de atención hospitalaria y de las de atención primaria, en las que se integran siete centros de salud. El laboratorio programado recibe una media de 700 analíticas diarias entre las secciones de bioquímica básica, hormonas, marcadores tumorales, vitaminas, urianálisis y gastroenterología e inmunología, además de otras pruebas especiales. El laboratorio de hematología y el de microbiología se encuentran separados y tampoco serán objeto del presente estudio. Desde el año 2018 el laboratorio cuenta con certificado mediante la ISO 9001-2015, y se obtuvo un riesgo elevado en uno de los subprocesos: comunicación de valores críticos, la causa fue tener en vigencia un protocolo obsoleto (los valores críticos hasta ese momento se pueden consultar en la Tabla 1) y no consensuado con los clínicos. Al ser un riesgo clasificado como muy alto, la respuesta fue disminuir el riesgo mediante una actualización del protocolo en consenso con los clínicos.

\section{Para ello:}

Se realizó una encuesta (Tabla 2) por un equipo compuesto por facultativos del laboratorio. Se incluyeron datos del modo de aviso (teléfono, email, fax), de la persona a la que hay que avisar (médico, enfermera o administrativo), una pregunta para valorar la importancia que los clínicos le otorgan al aviso de valores críticos (cultura de seguridad), y se encuestaba por 25 magnitudes prefijadas, cada una con dos valores y una opción libre en el caso de que el clínico considerara otro valor fuera de las opciones que les presentábamos. Una vez confeccionada la encuesta se difundió a los médicos de atención especializada por email a través de la dirección médica, y a los de atención primaria a través de la dirección de continuidad asistencial. La encuesta se difundió vía online.

Una vez recibidos los resultados, se analizaron entre todos los facultativos del servicio de análisis clínicos. Se realizó una técnica de consenso Delphi para llegar a los resultados finales de aviso de valores críticos.

\section{RESULTADOS}

\section{Encuesta sobre valores críticos}

Se obtuvieron 57 respuestas a la encuesta. Se desconoce a cuántos clínicos llegó, pues al ser una encuesta online la difusión se realizó desde la dirección tanto de atención especializada como de atención primaria.

La muestra tiene una representación mayoritaria en medicina de familia con 35\% (n:20) de las respuestas, el resto fue de especialidades médicas, dentro de las cuales aparecen pediatría, medicina intensiva, medicina interna, psiquiatría, urología, digestiva, oncología, etcétera. 
Todas los profesionales que respondieron consideran importante el aviso de valores críticos, es decir 100\%.

En cuanto a quién es la persona a la que se debería avisar, la gran mayoría, $77 \%$ indica que al propio clínico peticionario, y en el caso de no estar disponible 10\% indica que al médico de guardia o a la enfermera del paciente, se destaca que $7 \%$ indica que debería comunicarse al administrativo del servicio o del centro de salud, el restante $6 \%$ no sabe o no contesta.

$\mathrm{Si}$ atendemos a cómo prefieren que se les notifique el valor crítico, 58\% prefiere la vía telefónica, 23\% el email, mientras que el restante $19 \%$ indica otras maneras de avisar, por ejemplo, el fax, a través del buscador, si está disponible, o a través de la propia historia electrónica).

En cuanto a los valores críticos per se, las respuestas obtenidas pueden observarse en la Tabla 3.

\section{Consenso Delphi entre los facultativos}

Se realizó un consenso entre los facultativos del laboratorio, basándonos en nuestro protocolo anterior, y en la encuesta. Mediante el consenso Delphi fueron necesarias tres reuniones para realizar un consenso por unanimidad. Los valores finalmente consensuados fueron los recogidos en la Tabla 1, junto con los valores críticos del protocolo previo. Además se acordó: el modo de proceder antes de avisar que no es objeto de este estudio (comprobación de la muestra, posibles interferencias preanalíticas y analíticas, comprobación del resultado por otro analizador, búsqueda de valores previos en el paciente, etcétera), el modo de aviso y a quién se avisa, todo ello se plasmó en un protocolo normalizado disponible también para los clínicos. Lo más relevante del protocolo se puede ver en la Tabla 4.

\section{Tabla 2: Encuesta a los clínicos.}

Perfil del clínico encuestado ¿A qué especialidad pertenece?

¿Años de experiencia?

Preguntas relacionadas con el aviso ¿Considera importante el aviso de valores críticos? ¿Qué vía considera la mejor para el aviso de valores críticos? ¿A quién se debe avisar?

Pruebas a valorar y valor crítico

Glucosa mg/dL: nivel bajo

Glucosa mg/dL: nivel alto

Sodio $\mathrm{mEq} / \mathrm{L}$ : nivel bajo

Sodio $\mathrm{mEq} / \mathrm{L}$ : nivel alto

Potasio $\mathrm{mEq} / \mathrm{L}$ : nivel bajo

Potasio $\mathrm{mEq} / \mathrm{L}$ : nivel alto

Creatinina mg/dL: nivel alto

Urea $\mathrm{mg} / \mathrm{dL}$ : nivel alto

Calcio* $\mathrm{mg} / \mathrm{dL}$ : nivel bajo

Calcio* mg/dL: nivel alto

Ácido úrico $\mathrm{mg} / \mathrm{dL}$ : nivel alto

Aminotransferasas U/L: nivel alto

Bilirrubina $\mathrm{mg} / \mathrm{dL}$ : nivel alto

Creatininkinasa U/L: nivel alto

Fósforo mg/dL: nivel bajo

Fósforo $\mathrm{mg} / \mathrm{dL}$ : nivel alto

Digoxina $\mathrm{ng} / \mathrm{mL}$ : nivel alto

Lactato deshidrogenasa U/L: nivel alto

Magnesio $\mathrm{mg} / \mathrm{dL}$ : nivel bajo

Magnesio $\mathrm{mg} / \mathrm{dL}$ : nivel alto

Troponina $\mathrm{pg} / \mathrm{mL}$ : nivel alto

TSH UI/mL: nivel alto

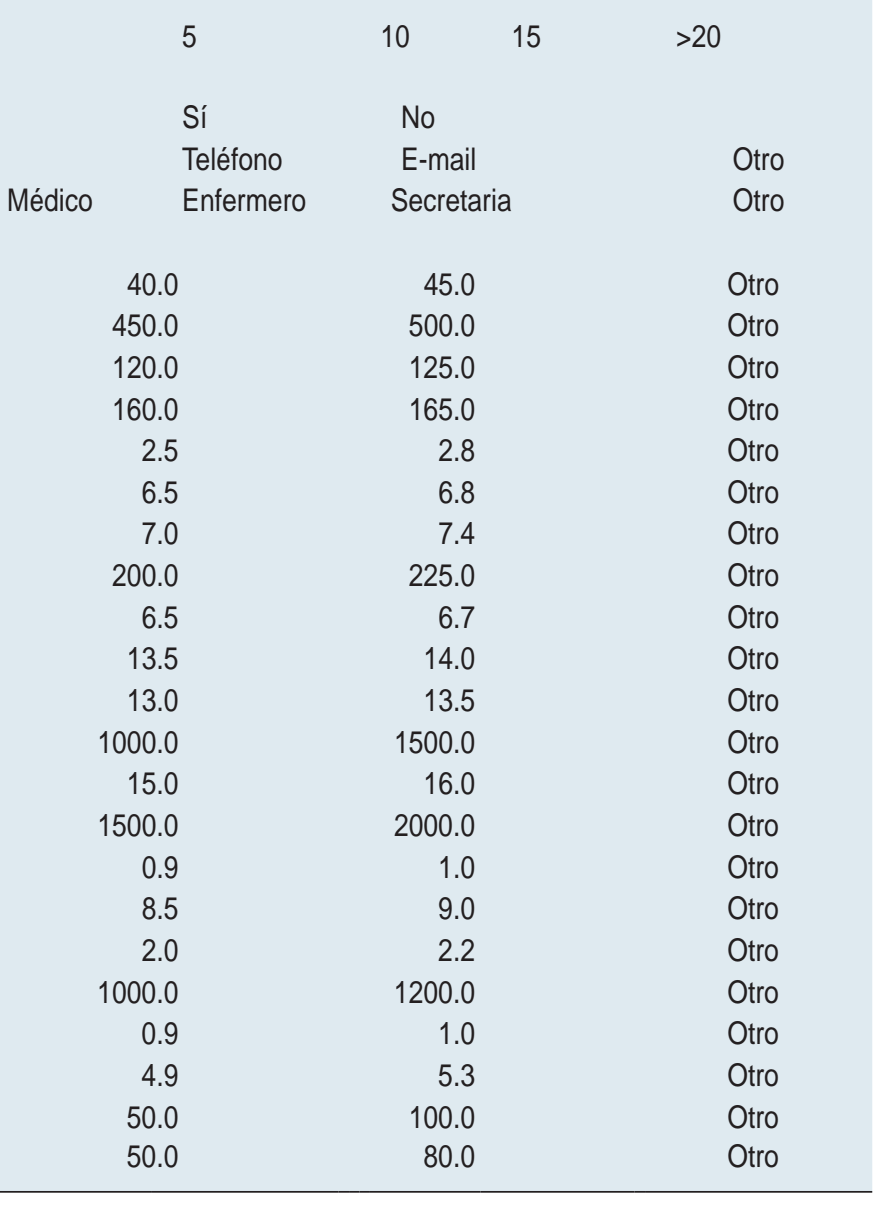




\section{Tabla 3: Respuestas a la encuesta: magnitud y valores.}

\begin{tabular}{|c|c|c|}
\hline Magnitud & Valor crítico & $\begin{array}{l}\text { Porcentaje de } \\
\text { respuestas }\end{array}$ \\
\hline \multirow[t]{2}{*}{ Hipoglucemia } & $<40 \mathrm{mg} / \mathrm{dL}$ & 19.6 \\
\hline & $<45$ mg/dL & 71.4 \\
\hline \multirow{2}{*}{ Hiperglucemia } & $>450$ mg/dL & 68.4 \\
\hline & $>500$ mg/dL & 10.5 \\
\hline \multirow[t]{2}{*}{ Hiponatremia } & $<120 \mathrm{mEq} / \mathrm{L}$ & 28.1 \\
\hline & $<125 \mathrm{mEq} / \mathrm{L}$ & 71.9 \\
\hline \multirow[t]{2}{*}{ Hipernatremia } & $>160 \mathrm{mEq} / \mathrm{L}$ & 78.6 \\
\hline & $>165 \mathrm{mEq} / \mathrm{L}$ & 12.5 \\
\hline \multirow[t]{2}{*}{ Hipopotasemia } & $<2.5 \mathrm{mEq} / \mathrm{L}$ & 42.1 \\
\hline & $<2.8 \mathrm{mEq} / \mathrm{L}$ & 56.1 \\
\hline \multirow[t]{2}{*}{ Hiperpotasemia } & $>6.5 \mathrm{mEq} / \mathrm{L}$ & 76.8 \\
\hline & $>6.8 \mathrm{mEq} / \mathrm{L}$ & 16.9 \\
\hline \multirow[t]{2}{*}{ Creatinina } & $>5 \mathrm{mg} / \mathrm{dL}$ & 67.9 \\
\hline & $>7 \mathrm{mg} / \mathrm{dL}$ & 10.0 \\
\hline \multirow[t]{2}{*}{ Urea } & $>200$ mg/dL & 72.7 \\
\hline & $>225$ mg/dL & 14.5 \\
\hline \multirow[t]{2}{*}{ Hipocalcemia } & $<6.5 \mathrm{mg} / \mathrm{dL}$ & 65.5 \\
\hline & $<6.7 \mathrm{mg} / \mathrm{dL}$ & 34.5 \\
\hline \multirow[t]{2}{*}{ Hipercalcemia } & $>13.5 \mathrm{mg} / \mathrm{dL}$ & 79.6 \\
\hline & $>14$ mg/dL & 18.5 \\
\hline \multirow[t]{2}{*}{ Ácido úrico } & $>13 \mathrm{mg} / \mathrm{dL}$ & 54.9 \\
\hline & $>13.5 \mathrm{mg} / \mathrm{dL}$ & 39.2 \\
\hline \multirow[t]{2}{*}{ Transaminasas } & $>1,000 \mathrm{U} / \mathrm{l}$ & 71.0 \\
\hline & $>1,500 \mathrm{U} / \mathrm{I}$ & 5.0 \\
\hline \multirow[t]{2}{*}{ Bilirrubina } & $>15$ mg/dL & 65.5 \\
\hline & $>16$ mg/dL & 3.0 \\
\hline \multirow[t]{3}{*}{ Creatininquinasa } & $>1,500 \mathrm{U} / \mathrm{L}$ & 64.3 \\
\hline & $>2,000 \mathrm{U} / \mathrm{L}$ & 16.1 \\
\hline & Otros: $1,000 \mathrm{U} / \mathrm{L}$ & 19.6 \\
\hline \multirow[t]{3}{*}{ Hipofosfatemia } & $<0.9 \mathrm{mg} / \mathrm{dL}$ & 28.6 \\
\hline & $<1 \mathrm{mg} / \mathrm{dL}$ & 71.4 \\
\hline & $>8.5 \mathrm{mg} / \mathrm{dL}$ & 76.4 \\
\hline Hiperfosfatemia & $>9$ mg/dL & 20.0 \\
\hline \multirow[t]{2}{*}{ Hipomagnesemia } & $<0.9 \mathrm{mg} / \mathrm{dL}$ & 40.0 \\
\hline & $<1 \mathrm{mg} / \mathrm{dL}$ & 60.0 \\
\hline \multirow[t]{2}{*}{ Hipermagnesemia } & $>4.9 \mathrm{mg} / \mathrm{dL}$ & 75.5 \\
\hline & $>5.3 \mathrm{mg} / \mathrm{dL}$ & 18.9 \\
\hline \multirow[t]{2}{*}{ LDH } & $>1,000 \mathrm{U} / \mathrm{L}$ & 72.2 \\
\hline & $>1,200 \mathrm{U} / \mathrm{L}$ & 20.4 \\
\hline \multirow[t]{2}{*}{ Digoxina } & $>2 \mathrm{ng} / \mathrm{mL}$ & 50.9 \\
\hline & $>2.2 \mathrm{ng} / \mathrm{mL}$ & 47.2 \\
\hline \multirow[t]{2}{*}{ Troponina } & $>50 \mathrm{pg} / \mathrm{mL}$ & 82.7 \\
\hline & $>100 \mathrm{pg} / \mathrm{mL}$ & 13.5 \\
\hline TSH & $>50 \mathrm{UI} / \mathrm{mL}$ & 79.6 \\
\hline TSH & $>70 \mathrm{UI} / \mathrm{mL}$ & 20.4 \\
\hline
\end{tabular}

\section{DISCUSIÓN}

Desde el laboratorio estábamos conscientes de que el protocolo previo no tenía en cuenta la opinión de los clínicos, cosa que hoy en día difiere de lo descrito en la bibliografía. $4,7,14,17$

La encuesta tiene un porcentaje de participación bajo pese a desconocer a cuántos clínicos llegó la encuesta, y pensamos que podría haber sido un punto mejorable en el estudio, si consideramos todos los clínicos de siete centros de salud de primaria y especializada, 57 respuestas son bastante escasas. Por todo esto hay que ir con especial cuidado con las respuestas. Si atendemos a la cultura de seguridad del paciente de la organización, pensamos que se podría medir con la pregunta: ¿Considera de utilidad el aviso de valores críticos? Ya que todos los clínicos que respondieron lo hicieron a esta pregunta con un sí, ¿podríamos afirmar que la cultura de seguridad es buena?, ¿qué están concientizados? Creemos que la baja participación en la encuesta (insistimos, sin saber el número real de los clínicos a los que les llega la encuesta) muestra todo lo contrario, no son conscientes de la importancia que tiene para la seguridad del paciente el correcto aviso de determinados valores críticos en ciertas magnitudes, conclusión concordante con lo publicado al respecto en la literatura. ${ }^{18}$ Debemos seguir trabajando para impregnar la cultura de la seguridad del paciente en la organización, pero se necesita un liderazgo que nos acompañe, pues sin él es del todo imposible.

La gran mayoría de las respuestas va a favor del aviso telefónico, coincidente con lo descrito en la literatura; ${ }^{15,16}$ es más rápido e inmediato, pero hay que asegurarse de hacer un adecuado read-back, ${ }^{17}$ es importante saber lo que entiende la otra persona al otro lado del teléfono. Por el contrario, el aviso por email puede tener la ventaja de que queda escrito y tenemos pruebas de haber realizado el aviso, pero por otro lado nunca tenemos la seguridad de que llega al clínico peticionario en el momento adecuado. Sin embargo, dejamos esta opción cuando no es fácil localizar al clínico a criterio del facultativo y el valor se acerca más a un valor de alerta que a un valor crítico.

En cuanto a los valores consensuados, pocos difieren de los del protocolo previo; sin embargo, el consenso añade credibilidad a esos valores a la vez que los respalda. Lo que se percibe de las respuestas de los clínicos es que siempre se señalan los valores más conservadores, por lo que podríamos pensar que se hace por medicina defensiva, una lacra en el mundo actual de la asistencia sanitaria. Como cambios más destacables en los valores se observan cambios creatinina, hiponatremia, CK, y glucosa.

La bibliografía recomienda que los valores críticos deben quedar reflejados en función de la edad y del sexo, ${ }^{13}$ 
Tabla 4: Aspectos más relevantes del protocolo final.

Antes de proceder al aviso desde el laboratorio se comprobarán las condiciones idóneas de la muestra por posibles interferencias, y del aparato en el que ha sido procesado, comprobándolo si es necesario. Se valorará además la existencia de valores previos similares en el paciente, valorando a criterio del facultativo si procede el aviso

- ¿Quién informa?

El facultativo responsable de la sección, o en su defecto el facultativo suplente o residente de la sección

- ¿A quién se informa y cómo?

Se informará al clínico peticionario de la analítica, o en su defecto a un médico de la misma especialidad vía telefónica o vía email. Si se realiza vía telefónica siempre se preguntará la identificación de la persona que toma el aviso y nos aseguraremos de que ha recibido correctamente la información (read-back)

En el caso de pacientes de atención primaria, si se avisa por email, se comunicará al buzón genérico de la secretaria del centro peticionario. En ese aviso vía email constará: nombre y apellidos del paciente, médico peticionario, día de la extracción y aviso del valor crítico En el caso de no poder localizar al clínico peticionario de ninguna manera y bajo ningún supuesto de los anteriores, bajo criterio del facultativo de laboratorio se podrá contactar con admisión de urgencias que localizará al paciente directamente para que acuda a urgencias

- ¿Qué se informa?

Identificación del paciente inequívoca (es decir, con número de petición, nombre y apellidos, médico solicitante y fecha de extracción) Se informa que existen valores analíticos críticos, y que el médico debe consultar la analítica completa en el ordenador.

en nuestro estudio al no responder muchos profesionales de pediatría, únicamente propusimos como mejora valores de TSH y transaminasas diferenciados para los niños menores de 14 años.

La realización del consenso intralaboratorio a través del método Delphi refuerza el resultado final y fomenta la comunicación entre el servicio, estableciendo por último un protocolo por escrito, lo que disminuirá considerablemente la variabilidad a la hora de actuar de los distintos facultativos en cuanto a la forma y modo de avisar o no un valor crítico. La comunicación mejora todas las estrategias implantadas de cara a la seguridad del paciente, y los protocolos hacen lo mismo con la variabilidad de la práctica clínica, minimizándola, y mejorando a su vez los aspectos básicos de la estrategia.

Como indicadores para la valoración y el correcto funcionamiento de la implantación de la estrategia se establecen los siguientes:

- Porcentaje de valores críticos avisados respecto al total de pacientes.

- Porcentaje de valores críticos avisados respecto al total de valores críticos.

- Porcentaje de valores críticos avisados en los que el clínico ejerce una acción en el paciente.

- Porcentaje de valores críticos avisados por email al no poder localizar al clínico y al considerar un valor de alerta más que un valor de alarma.

Consideramos que el laboratorio ha ejercido un papel de responsabilidad y de buen hacer implementando una estrategia o más bien actualizando una estrategia que ya estaba en vigor mediante el consenso con los clínicos. Además con ello ha fomentado la cultura de seguridad del paciente en el propio laboratorio y se ha hecho extensiva a los departamentos médicos. Y con todo ello hemos cumplido además uno de los objetivos estratégicos que aparecían en el plan de Seguridad del Paciente del Servicio Madrileño de Salud.

\section{REFERENCIAS}

1. Sciacovelli L, Plebani M. The IFCC Working Group on laboratory errors and patient safety. Clin Chim Acta. 2009; 404 (1): 79-85.

2. Sciacovelli L, Aita A, Padoan A, Pelloso M, Antonelli G, Piva E et al. Performance criteria and quality indicators for the post-analytical phase. Clin Chem Lab Med. 2016; 54 (7): 1169-1176.

3. Mc Cay L, Lemer C, Wu AW. Laboratory safety and the WHO World Alliance for Patient Safety. Clin Chim Acta. 2009; 404 (1): 6-11.

4. Piva E, Plebani M. Interpretative reports and critical values. Clin Chim Acta. 2009; 404 (1): 52-58.

5. Price CP. Editorial: Automated critical value reporting; a contribution to systematization of clinical care and the value of laboratory medicine. Clin Biochem. 2014; 47 (13-14): 1161-1162.

6. Lundberg GD. When to panic over abnormal values. MLO Med Lab Obs. 1972; 4: 47-54.

7. Salinas M, López-Garrigós M, Asencio A, Lugo J, Gutiérrez M, Flors $L$ et al. Alert value reporting: A new strategy for patient safety. Clin Biochem. 2013; 46 (3): 245-249.

8. Clinical and Laboratory Standards Institute (CLSI), Management of Critical- and Significant-risk Results 1st Edition CLSI Guideline. GP47. Wayne PA: Clinical and laboratory Standards Institute; 2015.

9. The Joint Commission, 2017 National Patient Safety Goals, Last access on February 1st, 2017. Available at: https://www. jointcommission.org/lab_2017_npsgs.

10. www.enac.es/c/document/library

11. Campbell CA, Georgiou A, Westbrook JI, Horvath AR. What alert thresholds should be used to identify critical risk results: a systematic review of the evidence. Clin Chem. 2016; 62 (11): 1445-1457. 
12. Don-Wauchope AC, Wang L, Grey V. Pediatric critical values: laboratory-pediatrician discourse. Clin Biochem. 2009; 42 (1617):1658-1661.

13. Piva E, Pelloso M, Penello L, Plebani M. Laboratory critical values: automated notification supports effective clinical decision-making. Clin Biochem. 2014; 47 (13-14): 1663-1668.

14. Lippi G, Mattiuzzi C. Critical laboratory values communication: summary recommendations from available guidelines. Ann Trans Med. 2016; 4 (20): 400.

15. Piva E, Sciacovelli L, Pelloso M, Plebani M. Performance specifications of critical results management. Clin Biochem. 2017; 50 (10-11): 617-621.

16. Valenstein PN, Wagar EA, Stankovic AK, Walsh MK, Schneider F. Notification of critical results: a College of American Pathologists
Q-Probes study of 121 institutions. Arch Pathol Lab Med. 2008; 132 (12): 1862-1867.

17. Lippi G, Giavarina D, Montagnana M, Luca Salvagno G, Cappelletti P et al; SIBioC (Italian Society of Clinical Biochemistry and Clinical Molecular Biology); SIMEL (Italian Society of Laboratory Medicine); CISMEL (Italian Committee for Standardization of Laboratory and Hematological Methods); Inter-associative Study Group on the Extra-Analytical Variability of Laboratory Testing. National survey on critical values reporting in a cohort of Italian laboratories. Clin Chem Lab Med. 2007; 45 (10): 1411-1413.

18. Don-Wauchope AC, Chetty VT. Laboratory defined critical value limits: how do hospital physicians perceive laboratory based critical values? Clin Biochem. 2009; 42 (9): 766-770. 